\title{
INCORPORACIÓN DE ESPAÑA AL ESPACIO EUROPEO DE EDUCACIÓN SUPERIOR: EL CASO DE UNA UNIVERSIDAD CATALANA
}

\section{Introducción}

El objetivo de este artículo es presentar algunos resultados relevantes de una investigación, llevada a cabo recientemente, sobre el proceso de incorporación al Espacio Europeo de Educación Superior (EEES) de una universidad española de la Comunidad Autónoma de Catalunya ${ }^{2}$.

\subsection{El Espacio Europeo de Educación Superior}

La construcción del EEES debe situarse en el marco de las reformas impulsadas por el proceso económico de globalización, pilotado políticamente por diversas instancias internacionales, como el Banco Mundial, la Organización Mundial del Comercio y la Organización para la Cooperación y el Desarrollo Económico (OCDE).

Las instituciones de educación superior de los distintos países están sometidas a las mismas presiones pero no siguen mecánicamente un proceso de homogeneización, puesto que los contextos locales tienen sus propias tradiciones y las políticas de los Estados también responden a la correlación de fuerzas de sus propios países. Así pues, cada país y cada universidad recontextualiza las directrices que provienen de las instancias internacionales de acuerdo con su realidad y posibilidades (Bernstein, 1998; Vaira, 2004).

2 Este artículo es una reelaboración de la conferencia dictada por J.M. Masjuan en la CUCAE de la Universidad de Guadalajara, el 7 de febrero de 2006, bajo el título "Los profesores de las universidades europeas frente al proceso de integración educativa". El equipo completo de investigación está formado por: Josep M. Masjuan, Helena Troiano, Guillem Sala y Cris Molins, profesores; Marina Elías, Nelson Paulus y Cecilia Peraza, becarios. Se puede consultar en la red un informe descriptivo global (Masjuan (Coord.), 2006). La investigación se ha llevado a cabo en una universidad que cuenta con más de 25.000 estudiantes y tiene una posición fuerte en investigación. 
En este sentido, por ejemplo, la situación no es la misma en México que en la mayoría de los países de la Unión Europea, entre ellos España. En el primer caso, la firma del Tratado de Libre Comercio entre EE.UU., Canadá y México ha supuesto un fuerte avance de las directrices de la mercantilización y privatización de la enseñanza superior (Marum, 2002). En el caso de España y otros países de la Unión Europea, en los cuales fracasó la negociación de la ronda del GATTS de 1999, las presiones hacia la privatización de la enseñanza superior no han conseguido los mismos resultados hasta el presente, debido a la importancia de la universidad pública y a la defensa por parte de la población del Estado de Bienestar.

El proceso de creación del EEES tiene su origen en la Declaración de la Sorbona, firmada por los ministros de educación de Francia, Italia, Alemania y Reino Unido en 1998, siguiendo la tradición de la Carta Magna de las Universidades Europeas de 1988.

En junio de 1999, 29 ministros de educación europeos firman la Declaración de Bolonia y crean el Espacio Europeo de Educación Superior, el cual no se limita a los Estados miembros de la Unión Europea, sino que está abierto a la incorporación de otros Estados europeos.

La mencionada declaración recomendó seis actuaciones:

- La promoción de la movilidad de estudiantes, profesorado, investigadores y personal técnico administrativo.

- La promoción de la cooperación entre universidades para asegurar la calidad, desarrollando criterios y métodos comparables.

- La adopción de un sistema fácilmente legible y comparable de titulaciones, mediante la implantación de un suplemento europeo al título, con vistas a favorecer la ocupabilidad (employability) y la competitividad internacional del sistema europeo de enseñanza superior.

- La adopción de un sistema basado fundamentalmente en dos niveles principales de estudios: grado y posgrado. El título de grado, de una duración mínima de tres años, debe tener un valor específico, tanto académico como laboral. El segundo nivel, de una duración máxima de dos años, da lugar a la obtención de un título de posgrado, tal como está sucediendo en muchos países europeos. 
- El establecimiento de un sistema común de contabilización del crédito, basado en el trabajo del estudiante (European Credit Transfer System, ECTS).

- La promoción de una necesaria dimensión europea en la educación superior.

Posteriormente, las declaraciones de Lisboa (2000), Praga (2001), Barcelona (2002) y Berlín (2003) reafirman las propuestas anteriores y añaden algunos aspectos, como la educación a lo largo de toda la vida, la necesidad de incorporar los estudiantes en la toma de decisiones y la promoción del atractivo de las universidades europeas en el contexto internacional. Se pretende que el año 2010 la reforma esté plenamente en funcionamiento.

\subsection{El proceso en España y Cataluña}

Se puede afirmar, en líneas generales, que la tendencia histórica de la universidad española ha sido la evolución desde un modelo burocrático de gestión hacia un modelo colegial, a partir de la legislación del gobierno del Partido Socialista Obrero Español (PSOE) en 1983, con la permanencia de algunos aspectos del modelo burocrático y la incorporación de otros del modelo empresarial o gerencialista, sobre todo a partir de la promulgación de la Ley Orgánica de Universidades (LOU, 2001) por parte del gobierno del Partido Popular (PP).

La norma legal citada introduce las reformas necesarias para la incorporación de España al EEES. Entre 2003 y 2006 se desarrollaron diversos aspectos de la ley mediante la publicación de sendos decretos. No obstante, el proceso de reestructuración está retrasado con relación a los países europeos vecinos, puesto que las universidades están a la espera de la publicación de la lista oficial de títulos y su duración.

Cataluña ${ }^{3}$ promulgó la Llei d'Universitats de Catalunya (2003), en la cual se adaptaron las normativas estatales a las propias necesidades y conveniencias políticas y, en este contexto, el Departamento de Universidades y Sociedad de la Información (DURSI) inició en 2004 un

3 Se trata de una de las comunidades autónomas del Estado español, de siete millones de habitantes, con un determinado nivel de autonomía política y administrativa en distintos campos, entre los cuales se encuentra el de las universidades. 
proyecto piloto para promover la adaptación de algunas titulaciones al EEES. Así se incorporaron a la prueba piloto 22 titulaciones de diplomatura e ingeniería técnica y 25 titulaciones de licenciatura e ingeniería superior ${ }^{4}$.

Las titulaciones que se incorporaron voluntariamente al plan piloto debieron realizar diferentes trabajos de adaptación durante el primer trimestre del curso 2005-2006: ajustar los planes de estudio vigentes a las propuestas europeas sin introducir cambios que vulnerasen la normativa estatal, definir los perfiles de las titulaciones y las competencias para ajustarse al espíritu profesionalizador de la reforma y preparar nuevas metodologías docentes compatibles con el nuevo sistema.

Cabe resaltar que la Agència per a la Qualitat del Sistema Universitari de Catalunya (AQU) asoció directamente los ECTS a una nueva metodología docente centrada en los aprendizajes de los estudiantes (AQU, 2003), aspecto que no está recogido explícitamente en los documentos oficiales.

\section{Marco teórico}

La investigación que sustenta este artículo parte de un marco teórico neoinstitucionalista, procedente principalmente de la economía institucionalista y de la ciencia política para el análisis de las políticas públicas (Scharpf, 1997; North, 1990; Troiano, 2001; Bergquist, 1992; de Miguel 1995), complementado con aportaciones concretas de otras procedencias (Lewin, 1956; Elton, 1990).

En el presente artículo se distinguen analíticamente dos momentos fundamentales del proceso de implementación de la reforma por parte de la universidad objeto de estudio: el proceso de toma de decisiones por parte de las facultades y escuelas y el nivel de puesta en práctica de los nuevos programas y métodos de enseñanza ajustados a los créditos europeos por parte del profesorado.

4 Las diplomaturas e ingenierías técnicas constan en la actualidad de tres años de duración, la mayoría de las licenciaturas son de cuatro años y las ingenierías superiores y algunas licenciaturas de cinco. 


\subsection{La fase de toma de decisiones}

Para analizar este proceso utilizamos el modelo de Scharpf (1997), que muestra diferentes modos de interacción entre actores y de toma de decisiones en distintos contextos institucionales, tal como se resume en la tabla siguiente:

Tabla 1: Tipos de contextos institucionales y modos de interacción según el modelo de Scharpf. Contextos institucionales 5 .

\begin{tabular}{|l|c|c|c|c|}
\hline Modos interacción y toma de decisiones & Anárquico & Redes & Asociación & Organización \\
\hline Acción unilateral & $\mathrm{X}$ & $\mathrm{X}$ & $\mathrm{X}$ & $\mathrm{X}$ \\
\hline Acuerdos negociados & $(\mathrm{X})$ & $\mathrm{X}$ & $\mathrm{X}$ & $\mathrm{X}$ \\
\hline Voto mayoritario & & & $\mathrm{X}$ & $\mathrm{X}$ \\
\hline Dirección jerárquica & & & & $\mathrm{X}$ \\
\hline
\end{tabular}

Según el autor, los modos de interacción son posibles en diferentes contextos institucionales, aunque funcionan de manera algo distinta en cada uno de ellos. De todas maneras, la diagonal principal nos indica el modo de interacción más característico: la acción unilateral en el campo anárquico, la negociación en las redes, el voto mayoritario en las asociaciones y la decisión jerárquica en las organizaciones.

Se espera que las universidades, organizadas de acuerdo con el modelo colegial, funcionen en un contexto institucional asociativo con mecanismos de decisión de voto mayoritario, tendiendo al consenso. En cambio, que las universidades, organizadas de acuerdo con el modelo gerencialista, lo hagan en un contexto institucional de organización, más semejante a una empresa mercantil y con mecanismos de decisión jerárquicos.

La literatura especializada se refiere frecuentemente tanto a la existencia de modelos mixtos en el nivel institucional como al uso de modos de interacción para la toma de decisiones que no serían los esperables en aquel contexto institucional (Birnbaum, 1988; Kezar, 2001; Trowler, 1998).

5 Scharpf amplía con las siguientes palabras los contextos institucionales: campos anárquicos e instituciones mínimas; redes y sistemas de decisión conjuntos; asociaciones y asambleas representativas; organizaciones burocráticas y Estado. 
Las preguntas que se plantean son las siguientes: ¿cómo se han tomado las decisiones? ¿Cuál ha sido el grado de implicación de los actores en el proceso de decisión, teniendo en cuenta los procedimientos, las tradiciones y las normas institucionales de la universidad?

Cuando el cambio obedece a un plan, como es el caso, una de las fuerzas importantes del mismo es la de los líderes de la organización, puesto que disponen de autoridad para empujar la organización hacia la dirección que se ha decidido previamente. En el caso de la universidad, tanto en el equipo de gobierno como de los centros académicos.

En la fase de toma de decisiones y una vez tomadas, antes de iniciar la implementación propiamente dicha, es importante tener en cuenta lo que Lewin (1956) denomina el "proceso de descongelación". En él los líderes deben impulsar el cuestionamiento de las prácticas tradicionales, facilitando que todos los actores de la organización las analicen críticamente, y eliminar obstáculos proporcionando a los actores los recursos necesarios ${ }^{6}$. La pregunta que orienta este análisis es la siguiente:

Los líderes de la organización, ¿han llevado a cabo un proceso de discusión y crítica de las prácticas anteriores en relación con las nuevas metodologías docentes y, al mismo tiempo, han facilitado los recursos necesarios para afrontar el cambio?

\subsection{La fase de implementación de la innovación}

La tabla siguiente muestra la relación entre las características de los actores y las del entorno institucional en el momento de la puesta en práctica de las decisiones tomadas. Witte (2003) integra en un esquema muy útil las aportaciones citadas de North y Scharpf. En nuestro caso concreto se refiere a las acciones del profesorado incorporando nuevas metodologías didácticas, condicionadas por los contextos institucionales.

6 Los otros momentos son el reemplazo o momento de introducción de la innovación y la recongelación, como mecanismo de refuerzo para evitar el retorno al punto inicial. 
Tabla 2: Relación entre las características de los contextos institucionales y las de los actores, según la propuesta de Witte ${ }^{7}$.

\begin{tabular}{|l|l|}
\hline $\begin{array}{l}\text { Las instituciones condicionan los actores a } \\
\text { través de: }\end{array}$ & Los actores se caracterizan por sus: \\
\hline Oportunidades (recursos y normas) & $\begin{array}{l}\text { Capacidades (capital cultural sobre nuevas } \\
\text { metodologías) }\end{array}$ \\
\hline Mapas mentales (cultura compartida) & $\begin{array}{l}\text { Percepciones (creencias sobre su valor y sobre } \\
\text { posibilidades de aplicación) }\end{array}$ \\
\hline Incentivos (económicos y/o simbólicos) & $\begin{array}{l}\text { Preferencias (motivaciones hacia la reforma de } \\
\text { los métodos didácticos) }\end{array}$ \\
\hline
\end{tabular}

Para situar este marco general en alguna pregunta concreta de investigación se han tenido en cuenta las propuestas de Cryer y Elton (1990), a partir de las cuales se han seleccionado de la tabla anterior las oportunidades e incentivos que ofrece la institución y las percepciones y preferencias del profesorado. Estas últimas no se limitan al propio interés individualista, sino que incluyen predilecciones normativas vinculadas a la función. Así pues, las preguntas concretas a las que se pretende aportar elementos de respuesta son: ¿cómo percibe el profesorado la aplicación de los ECTS en relación con la calidad docente? ¿Qué expectativas tiene de aplicarlos en un futuro próximo y cuáles son los costos y beneficios que aprecia en el proceso de cambio?

\section{Metodología}

Se seleccionaron 10 titulaciones de cuatro áreas temáticas. De éstas, dos iniciaron la reforma el curso 2003-2004 (Matemáticas y Relaciones Laborales), cuando todavía no se había propuesto la prueba piloto; cuatro el curso 2004-2005 (Humanidades, Sociología, Física e Informática). Las cuatro restantes aún no habían decidido su participación en la experimentación de la reforma (Historia, Administración de Empresas, Química y Telecomunicaciones).

Las fuentes de información de la investigación han sido: documentos públicos en el ámbito de la universidad y de facultades; entrevistas a tres cargos académicos de cada una de las titulaciones

7 Las expresiones entre paréntesis son de los autores del artículo para situar el concepto en la investigación concreta y facilitar la comprensión. 
seleccionadas (10 titulaciones y 28 entrevistas en total) ${ }^{8}$ y entrega y recogida personal de cuestionarios con preguntas cerradas y abiertas a una muestra del profesorado de las titulaciones (424).

Específicamente, para este artículo hemos utilizado la información procedente de las entrevistas a los cargos académicos, con el fin de contestar las preguntas relacionadas con el proceso de toma de decisiones. Para contestar las preguntas referidas al proceso de implantación de los ECTS nos hemos servido de las respuestas a tres preguntas procedentes del cuestionario a los profesores.

\section{Resultados}

\subsection{La fase de toma de decisiones}

La decisión inicial en el plano institucional fue tomada por el equipo de gobierno y se aprobó en el Consejo de Gobierno, órgano supremo colegiado de la universidad ${ }^{9}$.

La tabla siguiente resume el proceso de decisión en los centros sobre la participación en la Prueba Piloto ${ }^{10}$, que es el que nos interesa, y las principales razones manifestadas por los cargos académicos entrevistados a propósito de participar o no en la misma.

8 Cinco titulaciones pertenecen a dos facultades y dos a la misma escuela.

9 El consejo de Gobierno está formado por 65 miembros: el rector, el secretario general y el gerente; seis vicerrectores, seis decanos, dos profesores, un estudiante (nombrados por el rector); ocho decanos o directores de escuela, 13 directores de departamento (elegidos estamentalmente), 17 profesores, cinco estudiantes, dos funcionarios de administración y servicios (elegidos por el Claustro) y dos miembros del Consejo Social.

10 Cuatro titulaciones participan en la Prueba Piloto oficial y dos iniciaron una experiencia piloto promovida por la universidad un curso antes. 
Tabla 3: Las decisiones tomadas y su justificación en las diferentes titulaciones seleccionadas según los cargos académicos

\begin{tabular}{|c|c|c|}
\hline & Decisión & Justificación \\
\hline Humanidades & $\begin{array}{l}\text { El decanato. Pasa directamente a la } \\
\text { Comisión de Titulación. Facilitada } \\
\text { por el hecho de que intervienen } \\
\text { muchos departamentos, algunos } \\
\text { con experiencia en las nuevas } \\
\text { metodologías. }\end{array}$ & $\begin{array}{l}\text { La innovación es buena e } \\
\text { imparable: incorporación a Europa, } \\
\text { movilidad de estudiantes, visibilidad } \\
\text { en el contexto español. } \\
\text { El rectorado pretende dar una } \\
\text { imagen moderna de la universidad. } \\
\text { La reforma está relacionada con la } \\
\text { calidad, el cambio de metodologías. }\end{array}$ \\
\hline Historia & $\begin{array}{l}\text { El decanato decide que esta } \\
\text { titulación no participe en la prueba } \\
\text { piloto. }\end{array}$ & $\begin{array}{l}\text { Desinformación y oposición de } \\
\text { sectores del profesorado. }\end{array}$ \\
\hline R. Laborales & $\begin{array}{l}\text { Decisión del rectorado con la } \\
\text { coordinación de la titulación, } \\
\text { previamente a la prueba piloto. } \\
\text { Paso de trámite por la junta de la } \\
\text { Facultad de Derecho. }\end{array}$ & $\begin{array}{l}\text { Interés de prestigiar la titulación y } \\
\text { oportunidad. }\end{array}$ \\
\hline Sociología & $\begin{array}{l}\text { Impulsada por el decanato. } \\
\text { Discusión y aprobación en la junta } \\
\text { de facultad con la oposición de un } \\
\text { sector importante del profesorado } \\
\text { de Ciencia Política. }\end{array}$ & $\begin{array}{l}\text { El proceso es imparable y apuntarse } \\
\text { rápido aportará beneficios. } \\
\text { La incorporación a Europa } \\
\text { es interesante y la reforma } \\
\text { metodológica también se valora } \\
\text { positivamente. }\end{array}$ \\
\hline Ad. Empresas & $\begin{array}{l}\text { Decisión de no participar de } \\
\text { momento por parte del decano, a } \\
\text { partir de una reunión de todos los } \\
\text { decanos de España relacionada con } \\
\text { el proceso en curso de la reforma de } \\
\text { los planes de estudio. }\end{array}$ & $\begin{array}{l}\text { El proceso es positivo e imparable. } \\
\text { Interesa Europa, la movilidad de } \\
\text { estudiantes es positiva, la visibilidad } \\
\text { de la facultad aumentará. } \\
\text { Se han apuntado rápido las } \\
\text { titulaciones que tienen incentivos de } \\
\text { desesperación. } \\
\text { El papel de los pedagogos es } \\
\text { negativo, crean problemas y no los } \\
\text { resuelven. }\end{array}$ \\
\hline Matemáticas & $\begin{array}{l}\text { Consulta formal del decano a } \\
\text { los órganos colegiales. Cierta } \\
\text { contradicción entre los cargos } \\
\text { académicos para definir los pasos } \\
\text { del proceso de decisión. } \\
\text { Se decide afirmativamente antes de } \\
\text { la prueba piloto oficial. }\end{array}$ & $\begin{array}{l}\text { Necesidad de cambiar el plan de } \\
\text { estudios hacia la profesionalización, } \\
\text { para atraer más estudiantes. } \\
\text { Reforma metodológica para mejorar } \\
\text { el rendimiento. }\end{array}$ \\
\hline Física & $\begin{array}{l}\text { Decisión del decano previa consulta } \\
\text { formal a órganos colegiales. } \\
\text { Decisión positiva de entrar en la } \\
\text { prueba piloto. }\end{array}$ & $\begin{array}{l}\text { Necesidad de cambiar el plan de } \\
\text { estudios hacia la profesionalización, } \\
\text { para atraer más estudiantes. } \\
\text { Conciencia de la diferencia entre las } \\
\text { titulaciones y, en consecuencia, de } \\
\text { los resultados de las reformas. }\end{array}$ \\
\hline
\end{tabular}




\begin{tabular}{|l|l|l|}
\hline Química & $\begin{array}{l}\text { Decisión de no participar en la } \\
\text { prueba piloto. }\end{array}$ & $\begin{array}{l}\text { Cansancio por las reformas que "se } \\
\text { han quedado en el cajón". }\end{array}$ \\
\hline Informática & $\begin{array}{l}\text { El decano, a partir de la propuesta } \\
\text { del rectorado. Inicialmente estaba } \\
\text { prevista otra ingeniería pero el } \\
\text { decano de la escuela se decantó por } \\
\text { la Informática, que es su disciplina. }\end{array}$ & $\begin{array}{l}\text { El proceso es imparable y conviene } \\
\text { por el acercamiento a Europa, la } \\
\text { movilidad. } \\
\text { La reforma metodológica se } \\
\text { debe hacer pero despacio. Cierto } \\
\text { escepticismo en relación con los } \\
\text { cambios metodológicos. }\end{array}$ \\
\hline Telecomunicaciones & $\begin{array}{l}\text { El decano prefirió centrarse en } \\
\text { Informática. }\end{array}$ & $\begin{array}{l}\text { Titulación muy nueva y con mucho } \\
\text { profesorado joven no funcionario. }\end{array}$ \\
\hline
\end{tabular}

Como muestra el resumen, se trata de un proceso inicialmente top-down, pero dejando libertad a las titulaciones para incorporarse al mismo (bottom-up).

Las decisiones han sido tomadas por el decanato de los centros académicos a partir del acuerdo con las comisiones de cada titulación. En este sentido, participaron las titulaciones donde había un cierto acuerdo sobre la conveniencia de participar. En definitiva, la decisión real se tomó en un proceso de negociación política en el contexto de una universidad que funciona colegialmente. Este proceso de negociación se ha llevado a término entre el responsable del equipo de gobierno de la universidad y el decano de las titulaciones afectadas o el coordinador de titulación, por una parte, y en el nivel de centro entre el decano o coordinador de titulación y las comisiones de titulación o los coordinadores. Las entrevistas con los cargos académicos parecen indicar que los responsables tuvieron en cuenta las opiniones del profesorado influyente en los diferentes contextos.

Las afirmaciones anteriores no implican que no se respetaran los cauces normativos para la toma de decisiones, como las juntas de centro (facultad, escuela, sección), pero se puede constatar que en algunos casos ha sido más bien un trámite. Destaca como excepción la titulación de Sociología, puesto que se explicita que necesitó más de una reunión de la junta de facultad para decidir finalmente la incorporación de sus dos titulaciones: Ciencia Política y Sociología. La junta estaba dividida entre el profesorado de esta última más favorable a la reforma y un sector importante del profesorado de Ciencia Política, opuesto a ella. El mecanismo de la toma de decisiones por mayoría 
en el órgano competente resolvió formalmente las discrepancias, pero esto no garantiza que se traduzca automáticamente en un cambio en la manera de enseñar de los profesores afectados, sino que se puede esperar una adaptación al cambio más bien en la forma que en el contenido, al menos en el corto plazo (Elton, 2005), debido a la falta de consenso y a las posibilidades de acción unilateral del profesorado universitario.

Los cargos académicos destacan que, debido al calendario de incorporación que planteó la administración autonómica, las decisiones se tuvieron que tomar con cierta prisa y, por tanto, aceptaron participar las titulaciones que o bien tenían problemas que resolver o estaban implicadas en un proceso de reforma previamente o veían alguna oportunidad en su rápida incorporación. Las titulaciones que se incorporaron no plantearon en general algún conflicto manifiesto, aunque en la titulación de Ciencia Política se mantiene un cierto nivel de conflicto latente debido a que la decisión fue tomada por la facultad que contiene Sociología y Ciencia Política. Las titulaciones que hemos incorporado en el estudio y que no quisieron participar mostraron claramente las razones de su decisión.

En todo caso, resultó imposible establecer un diálogo general sobre la reforma entre el profesorado y otros agentes universitarios (estudiantes y personal de servicios), y generar un consenso mínimo sobre la bondad de la misma. Aunque el mismo rector acudió a los centros a explicar la conveniencia de la incorporación al EEES, las respuestas de los cargos académicos muestran claramente que no se consiguió información suficiente ni se concluyó adecuadamente un proceso de discusión y crítica de las metodologías docentes vigentes y de las propuestas por la reforma.

\subsection{La fase de implementación de los ECTS por parte del profesorado}

Además de depender del contexto general de la universidad, el proceso de implementación del nuevo sistema de créditos depende también de los contextos particulares de los centros y departamentos donde precisamente el profesorado interactúa, establece vínculos con 
otros profesores, adquiere compromisos y comparte experiencias de docencia e investigación. Se ha prescindido de este nivel en este artículo por razones de espacio, pero a través de los datos se ha constatado que no todas las titulaciones y departamentos funcionan de la misma manera y, por consiguiente, se trata de un aspecto fundamental para comprender los mecanismos sociales que explican los resultados en ámbitos superiores del sistema (Trowler, 2005; Masjuan \& Troiano, 2006). Así pues, se realiza solamente una primera aproximación global al tema que nos ocupa.

\section{Las percepciones del profesorado sobre los ECTS}

Para realizar esta prueba se ha construido una tipología del profesorado a partir del cruce de dos variables: la valoración que éste hace de la mejora en la calidad docente que supondrá el proceso de Bolonia y la percepción del grado de cambio en la docencia que piensa llevar a la práctica cada profesor en los próximos años. La tabla siguiente muestra los cuatro perfiles resultantes y el porcentaje de profesorado en cada uno de ellos.

Tabla 4: Tipología del profesorado según su actitud frente al cambio de docencia: valoraciones y perspectivas de cambio ${ }^{11}$.

Percepción valorada de la reforma

\begin{tabular}{|l|l|l|}
\hline $\begin{array}{l}\text { Preferencias en relación con } \\
\text { el cambio docente. }\end{array}$ & $\begin{array}{l}\text { La aplicación del proceso } \\
\text { de Bolonia mejorará la } \\
\text { calidad docente. }\end{array}$ & $\begin{array}{l}\text { La aplicación del proceso } \\
\text { de Bolonia no mejorará la } \\
\text { calidad docente. }\end{array}$ \\
\hline $\begin{array}{l}\text { En los próximos cinco años } \\
\text { cambiará mi manera de } \\
\text { enseñar. }\end{array}$ & $\begin{array}{l}\text { Cambio positivo } \\
\text { Cambio negativo } \\
27 \%\end{array}$ \\
\hline $\begin{array}{l}\text { En los próximos cinco años } \\
\text { no cambiará mi manera de } \\
\text { enseñar }\end{array}$ & $\begin{array}{l}\text { Resistencia positiva } \\
14 \%\end{array}$ & $\begin{array}{l}\text { Resistencia negativa } \\
22 \%\end{array}$ \\
\hline
\end{tabular}

$\mathrm{N}=286 . \mathrm{N} . \mathrm{C} 33 \%$

11 Utilizamos el concepto de actitud porque resume muy bien la percepción valorada de la reforma y la preferencia práctica. Las preguntas son las siguientes: ¿qué valoración hace los aspectos relacionados con la Declaración de Bolonia en relación con la calidad de la enseñanza? (Muy positivo y Bastante $=$ Positivo; Indiferente, Bastante y Muy negativo = Negativo). ¿Cree que de aquí a cinco años habrá cambiado muchas cosas en su manera de enseñar? (Escala de cuatro posibilidades agrupadas en dos). 
Los datos muestran que el profesorado está poco informado y muy dividido. La moda de la distribución corresponde al profesorado que valora el cambio positivamente y considera que su manera de enseñar cambiará en el próximo futuro, pero se trata de una frecuencia muy baja, sobre todo teniendo en cuenta que un tercio del profesorado no tiene una opinión formada. La categoría etiquetada como "resistencia positiva" presenta dificultades de interpretación, puesto que mezcla los profesores que valoran el cambio positivamente pero no ven posible llevarlo a la práctica, ya sea por su edad o su dedicación a la investigación, con los que no piensan cambiar precisamente porque ya realizan una docencia más activa.

Razones aducidas a propósito de los costos y beneficios Las percepciones sobre costos y beneficios se han recabado a partir de una pregunta abierta, de la cual se obtuvieron 302 respuestas que han sido clasificadas en cuatro categorías, después de excluir 32 respuestas totalmente dispersas. Dos categorías se refieren a beneficios: mejor formación de los alumnos (27\%) y más satisfacción personal (14\%), y dos a los costos: peor formación de los alumnos (7\%) y más dedicación sin reconocimiento (51\%). Cada uno de los cuatro discursos recoge todos los matices expresados en conjunto, sin tener en cuenta el número de veces que han sido citados (Boudon, 2005, Manzo, 2005). Los resultados de este proceso de construcción se recogen a continuación. 
Tabla 5: Percepciones del profesorado sobre costos y beneficios de la aplicación de los ECTS (\% sobre el total de percepciones indicadas).

- Mejor formación de los estudiantes (27\%)

- El nuevo sistema permitirá mejorar la docencia, siempre que se haga bien, consensuando con otros profesores los programas, la materia, seleccionando contenidos, conociendo mejor los resultados, entre otros aspectos este es el camino a seguir más coherente con el mundo actual.

- Espero que este esfuerzo programador dará sus frutos en un aprendizaje más objetivo y claro, más eficaz, y proporcionará a los alumnos conocimientos más prácticos y útiles de cara el futuro. El cambio invita a escoger contenidos más interesantes, a replantearse los contenidos y a renovarlos, ya que la reflexión sobre las metodologías también facilita la reflexión sobre los contenidos y sobre las habilidades que deben manejar.

- Los nuevos métodos comportarán más dedicación de los estudiantes y aprenderán más. Las asignaturas serán más abiertas y se tendrán más en cuenta los conocimientos que interesen a los estudiantes.

- Aumentará la proximidad alumno-profesor y mejorará la participación y la implicación de los estudiantes. Comporta un seguimiento personalizado, lo cual es un reto no siempre fácil de realizar.

- Hacer la enseñanza más activa mejorará la dinámica de las clases y el ambiente de trabajo será más positivo, mejorando el interés de los estudiantes por la materia.

- Mejorará la percepción sobre el profesorado, puesto que seremos vistos más como asesores que como transmisores de contenidos.

- Más satisfacción profesional (14\%)

- Un proceso de enseñanza más satisfactorio que me va a permitir experimentar nuevas metodologías de enseñanza aprendizaje, y esto siempre es positivo cuando te gusta la docencia. También me va a permitir buscar nuevas fuentes de información para los alumnos y revisar rutinas, lo cual comporta pequeñas mejoras que repercutirán en un mejor aprendizaje de los estudiantes. Creo que el sistema que se propone puede mejorar el proceso de aprendizaje y, por tanto, la satisfacción profesional del docente, puesto que el contacto directo con los estudiantes alrededor de un proyecto práctico es mucho más enriquecedor y motivador para todos, también para los profesores.

- En definitiva, me beneficia moralmente, porque el rendimiento social de mi trabajo aumentará y en conjunto es un reto que puede generar mucha satisfacción.

- Mejor visibilidad y reconocimiento de la innovación en el aula y más satisfacción porque veo que los estudiantes lo valoran.

- Peor formación de los estudiantes (7\%)

- La reforma va a comportar un descenso del nivel de conocimientos y habría que pensar cómo compensarlo. La homogeneización puede ser contraproducente, ya que los conocimientos específicos y avanzados, fruto de la investigación, disminuirán.

- Con tanto portafolio, los estudiantes dejarán de consultar las fuentes originales (revistas, bases de datos). Algunas asignaturas se convertirán en marías, a partir del aumento de trabajo autónomo de los estudiantes. La asignatura como tal desaparecerá.

- Pienso que la obsesión por cuantificar el trabajo del estudiante va en detrimento de su esfuerzo. En general, esta metodología sólo se corresponde con una parte de los estudiantes, va a suponer un aumento del control y comporta riesgos psicosociales.

- Esta metodología hace creer al estudiante que todo es obligación y que todo se lo han de dar hecho. Una carrera es esfuerzo, trabajo, estudio.

- El aumento de horas de corrección hará disminuir el contacto con los estudiantes, la tutoría con tantos alumnos no es eficaz. En fin, una reforma a costo cero es perjudicial para alumnos y profesores.

- Haría falta saber cómo se han valorado los ECTS en otros países que tienen experiencia, ya que hay informes negativos. No siempre un cambio significa mejora. 
- Más dedicación sin reconocimiento (51\%)

- La nueva metodología comporta más trabajo sin una compensación económica ni reconocimiento; es un voluntarismo. Exige programar la materia minuciosamente, hacer esfuerzos de adaptación, repensar y reestructurar las asignaturas, preparar las clases, planificar actividades, más dedicación docente, tutorizar personalmente a los alumnos, más trabajo de evaluación, horarios más complicados, gestión más difícil de la agenda personal, necesidad de dedicar tiempo a la formación.

- Es desmotivador convertirse en corregidores de ejercicios y supone mucho trabajo sin saber si los planes de estudios sobre los que aplicamos el cambio tendrán continuidad.

- Si no se reduce el número de alumnos por grupo es muy difícil, ya que es un sistema pensado para grupos reducidos. Perderemos flexibilidad, ya que aumentan los aspectos administrativos de la docencia en lugar de mejorar la preparación temática. Hacer más papeles representa menos tiempo para leer. Hay mucho trabajo que considero inútil, sobre todo si interviene el IDES.

- Perjudica, en tanto que es tiempo que no se puede dedicar a la investigación y cada día es más importante y hay más presión directa en esta dirección. Además, la investigación está mejor considerada y el cambio puede afectar negativamente a la estabilización y promoción del profesorado joven.

- El tiempo dedicado a los estudiantes quema mucho porque están poco motivados y no conseguiremos nada para mejorar su preparación. Requerirá más dedicación "oscura", más horas de preparación que no tengo claro que se la merezcan. Costará cambiar la dinámica actual del estudiante pasivo.

- No me parece que la docencia pueda cambiar mucho si no se ponen más recursos materiales y humanos. Faltan espacios adecuados para trabajar cómodamente, faltan profesores ayudantes.

- Me parece que muchos profesores no lo ven así y costará mucho que se valore el esfuerzo de quienes lo hacen.

En la tabla se puede observar que:

- Los costos y beneficios han sido percibidos principalmente en términos normativos, es decir, en relación con el ejercicio del propio rol, aunque también comporten aspectos relacionados con el propio interés.

- El análisis detallado de los discursos del profesorado confirma que no se da una oposición frontal hacia las nuevas metodologías docentes.

- La falta de recursos humanos para aminorar la exigencia de más dedicación aparece como un aspecto muy relevante, constatado también a partir de otras preguntas del cuestionario.

\section{Hipótesis fundada de cuatro perfiles típicos de profesorado}

Por último, la tabla siguiente es una construcción a partir de los cuatro perfiles de profesorado mostrados en la tabla 4, ampliando las respuestas a la pregunta cerrada sobre la valoración de la reforma para la mejora de la docencia con las razones sobre los costos y beneficios expuestos en la tabla 2. 
Tabla 6: Hipótesis sobre las razones que justifican los cuatro tipos de profesorado existentes.

\begin{tabular}{|c|}
\hline $\begin{array}{l}\text { Cambio positivo (38\%): } \\
\text { El nuevo sistema de enseñanza ofrece mejor formación a los estudiantes y, aunque a } \\
\text { corto plazo me va a pedir más dedicación sin reconocimiento, al final el cambio me } \\
\text { dará más satisfacción profesional. }\end{array}$ \\
\hline $\begin{array}{l}\text { Cambio negativo ( } 27 \%) \text { : } \\
\text { El nuevo sistema de enseñanza ofrece peor formación a los estudiantes y, además, me } \\
\text { va a pedir más dedicación sin compensación; de todas maneras, no tengo más remedio } \\
\text { que cambiar porque me lo exigen. }\end{array}$ \\
\hline $\begin{array}{l}\text { Resistencia negativa (22\%): El nuevo sistema de enseñanza ofrece peor formación a los } \\
\text { estudiantes y, además, me pide mayor dedicación sin compensación, por consiguiente, } \\
\text { no pienso cambiar mi manera de enseñar. }\end{array}$ \\
\hline $\begin{array}{l}\text { Resistencia positiva (14\%): a) El nuevo sistema de enseñanza ofrece mejor formación a } \\
\text { los estudiantes y hace tiempo que practico una enseñanza más activa, por tanto, no } \\
\text { tengo que cambiar nada fundamental. b) El nuevo sistema de enseñanza ofrece una } \\
\text { mejor formación a los estudiantes, pero me exige más dedicación y a estas alturas no } \\
\text { puedo cambiar. }\end{array}$ \\
\hline
\end{tabular}

Siguiendo este modelo resumido y ampliándolo con las razones extensas transcritas anteriormente se consigue una buena aproximación a la racionalidad subjetiva contextualizada del profesorado, la cual explica su posición frente al cambio.

De la interacción entre el profesorado de los cuatro perfiles en sus entornos concretos (micronivel) dependerá principalmente el resultado del proceso del conjunto de la universidad; es decir, cuál será el modelo de docencia que acabará imponiéndose como legítimo, tanto en el discurso como en la práctica, reorganizando el conjunto del sistema en el nivel superior (macronivel). En el futuro, el sistema resultante ejercerá presión cultural y coactiva sobre el profesorado restante y de nueva incorporación en la dirección, de la conformidad con la nueva situación (Haken, 1988).

Desde el punto de vista político, el análisis detallado de los discursos del profesorado ofrece muchos elementos a los gestores del cambio para modificar aquellos aspectos que pueden hacer fracasar la experiencia iniciada. 


\section{Discusión de resultados y conclusiones}

Un primer aspecto a tener en cuenta en la valoración de los resultados obtenidos es que los datos se recogieron durante el inicio de la implementación de la reforma europea; por consiguiente, es imposible obtener resultados consolidados que se puedan dar por definitivos. Se trata fundamentalmente de una primera aproximación a cómo afronta el proceso de cambio la universidad objeto de estudio.

A continuación se responde a las preguntas planteadas, añadiendo algunas reflexiones muy breves de carácter político.

1. En una universidad de fuerte tradición colegial, las decisiones en torno a la participación de las titulaciones en la prueba piloto siguieron en la práctica un modelo de negociación política, aunque se respetasen formalmente los mecanismos institucionales de decisión. Solamente en caso de conflicto entre los profesores, los órganos colegiales sirvieron como lugar de discusión de las ventajas e inconvenientes y, finalmente, para tomar la decisión pertinente a partir del voto mayoritario. Este sistema de toma de decisiones no ha conseguido generar un consenso previo generalizado en torno a la conveniencia de la reforma y sobre las condiciones necesarias para llevarla a cabo.

2. El proceso de "descongelación" recomendado por Kurt Lewin para garantizar los cambios en las organizaciones complejas no se ha realizado adecuadamente, principalmente por las prisas en su implementación. El riesgo principal es que el cambio en la docencia acabe siendo puramente formal, es decir en el método de contar los créditos. Este resultado puede realimentar las posiciones contrarias a la reforma de la metodología docente y tener como consecuencia que en el futuro sea más difícil realizar una docencia más activa, basada en los aprendizajes de los estudiantes (Senge, 2002).

3. El profesorado está poco informado y muy dividido en relación con el cambio docente. De la acción de los líderes facilitando el proceso y, sobre todo, de las interacciones entre el profesorado en los diferentes contextos disciplinares y docentes dependerá el futuro de la reforma, cuyo éxito en el momento actual no puede darse por descontado. 
4. El profesorado asocia los beneficios de la reforma en la metodología docente con la mejora en la calidad de la docencia y la satisfacción profesional que se derivará de la misma, aun considerando que los costos en dedicación serán elevados. En cambio, el rechazo a la reforma está asociado a la valoración negativa de la misma desde el punto de vista docente y a los costos humanos de su implementación. Parece pues un elemento fundamental incrementar o redistribuir los recursos humanos existentes.

\section{Agradecimientos}

Esta investigación forma parte del Plan Nacional de Investigación Científica, Desarrollo e Investigación Tecnológica (BSO2003-06395/CPSO) y ha sido financiada por el Ministerio de Educación y Ciencia y FEDER.

\section{Referencias bibliográficas}

Agència per a la Qualitat del Sistema Universitari de Catalunya. (2003) Marc General per a la Integració Europea. Barcelona: AQU.

Bernstein, B. (1998) Pedagogía, control simbólico e identidad. Madrid: Morata.

Bergquist, W.H. (1992) The Tour Cultures of the Academy. San Francisco: Jossey Bass.

Birnbaum, R. (1988) How Colleges Work. London: Jossey Bass Publishers.

Boudon, R. (2005) Raisons, bonnes raisons. Paris: P.U.F.

Cryer, P. and Elton, L. (1990) Catastrophe theory: A unified model for educational change. Studies in Higher Education, 15-1, pp. 75-86.

De Miguel, M. (1995) La evaluación de las instituciones universitarias y la toma de decisiones. En Arbizu, F. (ed.) La toma de decisiones. Unidad de Evaluación, ICE-Universidad del País Vasco, pp. 77-142.

Elton, L. (2005) Recent Developments in Student Learning in Britain and their relationship to the Bologna Declaration. Papers, 76, pp. 33-45.

Haken, H. (1988) Fórmulas de éxito en la naturaleza. Barcelona: Salvat.

Kezar, A.J. (2001) Understanding and Facilitating Organizational Change in the $21^{\text {st }}$. Century. Recent Research and Conceptualisations. Higher Education Report, 28(4).

Lewin, K. (1956) La teoría del campo en la ciencia social. Barcelona: Paidós.

Masjuan, J.M. y Troiano, H. (2006) El proceso de implementación de los ECTS 
en una universidad catalana. Pendiente de aceptación por parte de la Revista española de investigaciones sociológicas.

Masjuan, J.M.; Troiano, H.; Sala, G.; Molins, C.; Elias, M.; Paulus, N. y Peraza, C. El profesorat d'una universitat catalana davant de la incorporació a l'EEES. Web site: http://selene.uab.es/fxmiguel/GRET/

Marum, E. (2002) Repercusiones del TLCAN en la formación de recursos humanos en México. En: Preciado, J.; Rocha, A. y Marum, E. (coord.) Dinámicas y escenarios estratégicos de la integración en América Latina. pp.169-180.

Manzo, G. (2005) Variables, mécanismes et simulations: analyse critique des trois méthodes. Revue Française de Sociologie, 46(1), pp. 37-74.

North, D.C. (1990) Institutions, Institutional Change and Economic Performance. Cambridge: Cambridge University Press.

Scharpf, F.W. (1997) Games Real Actors Play. Boulder (Colorado): Westview Press.

Senge, P. (2002) La quinta disciplina. Barcelona: Granica.

Troiano, H. (2001) La reforma universitària: gestió del pas d'un entorn institucional a un entorn parcialment tècnic. Educar, 28, pp. 163-178.

Trowler, P. (1998) What Managerialist Forget: higher education credit frameworks and managerialist ideology. Internacional Journal in Sociology of Education, 8(1), pp. 91-110.

Trowler, P. (2005) A Sociology of Teaching, Learning and Enhancement: improving practices in higher education. Papers, 76, pp. 13-32.

Vaira, M. (2004) Globalisation and higher education organizational change: A framework for analysis. Higher Education, 48, pp. 483-510.

Witte, J. (2003) A theoretical framework for an international comparative study of the introduction on two/tier study structures in the context of the Bolonia Process. Paper presented to the CHER Conference, 4-6 September, 2003, Porto.

Recibido: 4 de agosto de 2009

Aceptado: 5 de octubre de 2009 